\title{
Reste eines Elasmosauriers aus der Oberkreide von Schleswig-Holstein in der Sammlung des Naturkunde-Museums Bielefeld
}

\author{
Sven SACHS, Bielefeld / Engelskirchen \\ Joachim LADWIG, Norderbrarup
}

Mit 4 Abbildungen

Inhalt

Seite

1. Einleitung 29

2. Fundort 30

3. Fundgeschichte 31

4. Systematische Paläontologie 31

5. Diskussion 33

6. Danksagung 34

7. Literatur 34

\section{Zusammenfassung}

Im Sommer 2016 erhielt das Naturkunde-Museum Bielefeld diverse Reste eines Elasmosauriers aus dem Obercampanium von Kronsmoor (Kreis Steinburg) in Schleswig-Holstein. Das Material umfasst fragmentarische Wirbel und Phalangen sowie nicht näher bestimmbare Fragmente. Weitere Teile des gleichen Skeletts (ein Zahn, Cervical-, Dorsal- und Caudalwirbel, Teile der Gliedmaßen und 110 Gastrolithen) befinden sich in der Sammlung des Geologischen Instituts der Universität Hamburg sowie in Privatsammlungen.

\begin{abstract}
In 2016, the Natural History Museum Bielefeld received fragmentary remains of an elasmosaurid plesiosaurian from the upper Campanian of Kronsmoor (Steinburg district) in Schleswig-Holstein (northern Germany). The material includes incomplete vertebrae, phalanges and unidentifiable fragments. Additional parts of the same skeleton (a tooth, cervical-, dorsal- and caudal vertebrae, limb elements and 110 gastroliths) are housed at the Institute for Geology of the University of Hamburg and in private collections.
\end{abstract}

\section{Verfasser:}

Sven Sachs, Naturkunde-Museum Bielefeld, Adenauerplatz 2, 33602 Bielefeld \&

Im Hof 9, 51766 Engelskirchen, E-Mail: Sachs.Pal@gmail.com

Joachim Ladwig, Pastoratstoft 1, 24392 Norderbrarup 


\section{Einleitung}

Elasmosauriden gehören in die Gruppe der Plesiosauria und waren aquatische Reptilien, die zumeist einen extrem verlängerten Hals besaßen, der aus bis zu 75 einzelnen Wirbeln bestehen konnte (SACHS \& KEAR 2015). Ihre Reste wurden auf allen Kontinenten entdeckt, doch während zum Beispiel aus den USA und Marokko nahezu vollständige Skelette vorliegen (siehe z.B. LOMAX \& WAHL 2013, WeLLES 1943) sind Funde in der europäischen Oberkreide eher fragmentarischer Natur. Speziell in der Oberkreide Deutschlands sind Elasmosaurierreste sehr selten. Beschrieben wurden bisher Zähne aus dem Oberturonium von Dresden in Sachsen (SACHS et al. 2016a, 2017a), Kieferfragmente und Zähne aus dem Untercampanium von Sehnde in Niedersachsen (FreRICHS 1994, SACHS 2011), ein Zahn aus dem Untercampanium von Bottrop

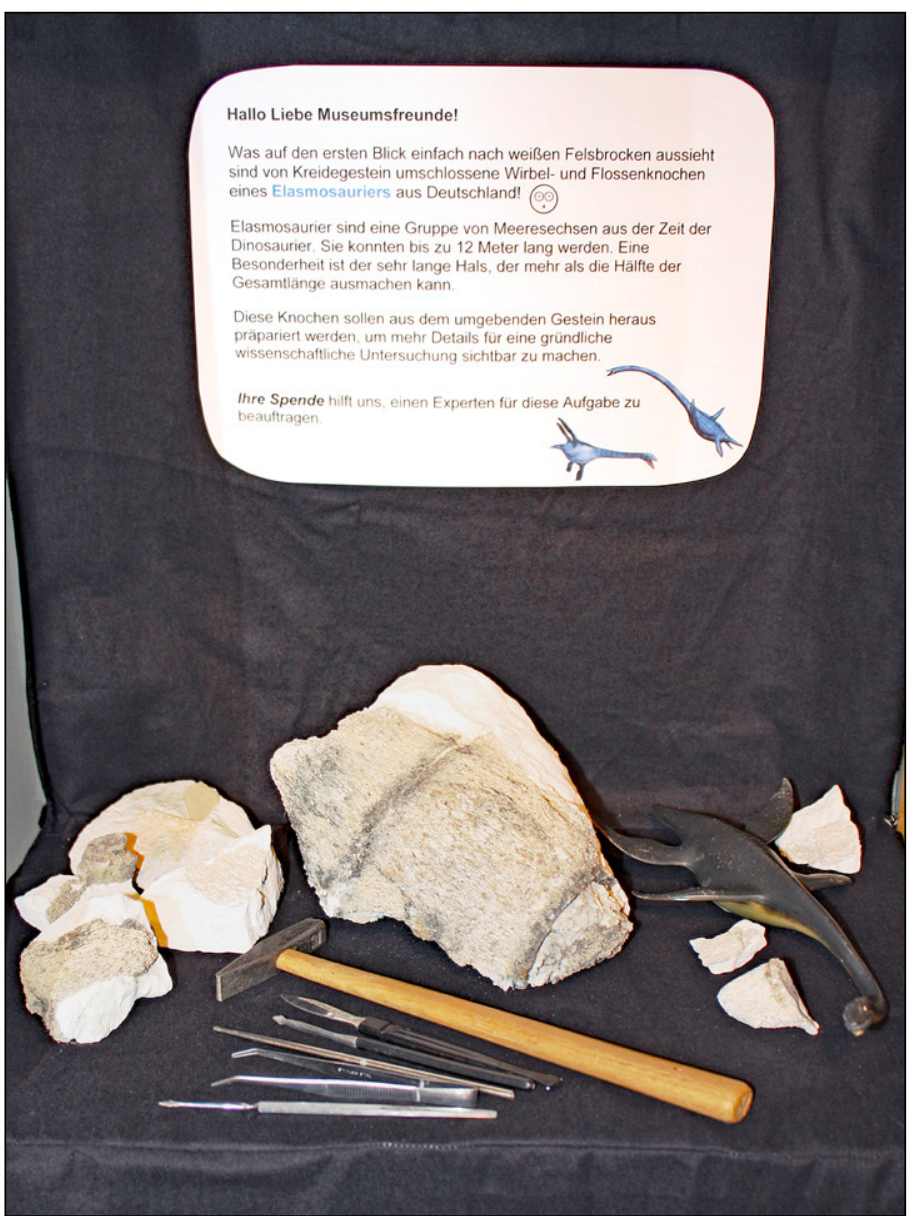

Abb. 1: Ausstellung der Exponate im Eingangsbereich des NaturkundeMuseums im Sommer 2016, um Spenden für die Präparation zu sammeln. in Nordrhein-Westfalen (SACHS et al. 2017b) sowie ein Dorsalwirbel aus dem oberen Untercampanium oder unteren Obercampanium von Wismar in MecklenburgVorpommern, der jedoch ein Geschiebefund ist und seinen Ursprung vermutlich im Gebiet des heutigen Südschwedens hat (Fотн et al., 2011). Der bisher vollständigste Fund dieser Gruppe aus der Oberkreide Deutschlands ist ein Skelett aus dem Obercampanium von Kronsmoor in Schleswig-Holstein, zu dem auch die hier beschriebenen Reste gehören. Diese hat der Zweitautor, Joachim Ladwig, dankenswerter Weise im Sommer 2016 dem Naturkunde-Museum Bielefeld übergeben. $\mathrm{Zu}$ diesem Zeitpunkt waren die Stücke noch

weitestgehend unpräpariert und konnten mithilfe von Spendengeldern (Abb. 1) im Herbst 2016 durch Paul Freitag aus Rostock fachgerecht nachpräpariert werden. In einem populärwissenschaftlichen Artikel stellte LADWIG (1997) die Fossilien bereits vor, sprach sie aber als Mosasaurierreste an. Weitere Teile des gleichen Individuums (siehe Fundgeschichte) wurden bereits von MAISCH \& SPAETH (2004) beschrieben. Sie befinden sich in der Sammlung des Instituts für Geologie der Universität Hamburg sowie in mehreren Privatsammlungen und umfassen einen Zahn, Cervical-, Dorsal- und Caudalwirbel, Teile der Gliedmaßen sowie 110 Gastrolithen. 


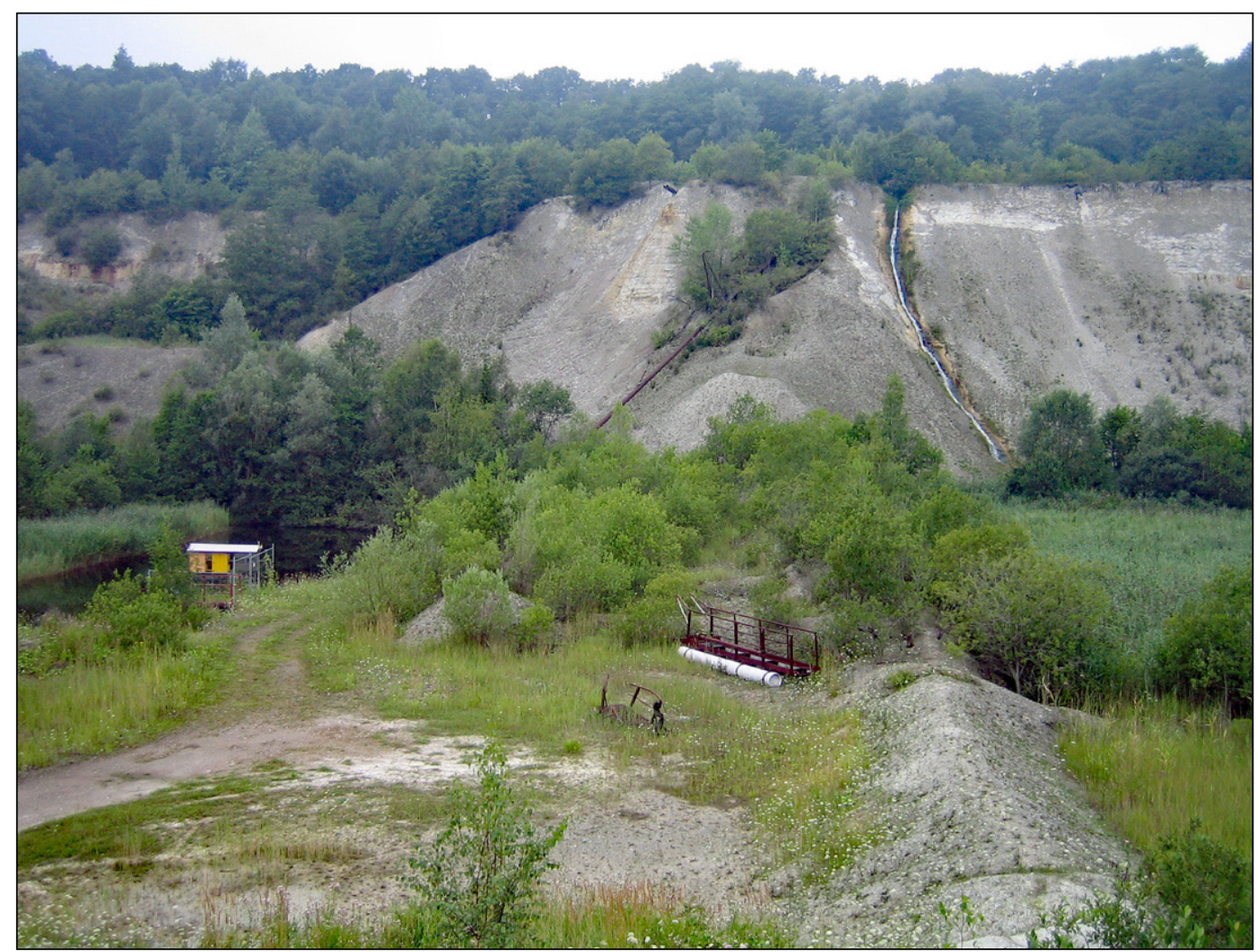

Abb. 2: Blick auf die Fundstelle an der Westwand der Grube "Saturn". Die Elasmosaurierreste lagen auf mittlerer Höhe des Hangs, rechts neben den Büschen (jetzt von Hangschutt bedeckt).

\section{Fundort}

Die Elasmosaurierreste wurden in der Schreibkreidegrube "Saturn" entdeckt (Abb. 2). Diese befindet sich in der Nähe des Dorfes Kronsmoor, wenige Kilometer südöstlich von Itzehoe in Schleswig-Holstein. Die Grube hat eine Länge von etwa einem Kilometer und eine Breite von ca. 700 Meter und ist seit 2002 stillgelegt. Aufgeschlossen sind hier die Schichten der oberen Oberkreide, die das Obercampanium (langei-Zone und grimmensis-/granulosus-Zone) und das Untermaastrichtium (lanceolata-, pseudobtusa-, obtusaund sumensis-Zone) umfassen (SCHULZ, 1985). Der Elasmosaurierfund wurde im mittleren Bereich der grimmensis-/granulosus-Zone an der Westwand der Grube "Saturn" entdeckt.
Hier ist, im Gegensatz zu den meisten anderen Teilen der Grube, eine hohe Funddichte der Echiniden Micraster grimmensis NIETSCH, 1921, Cardiaster granulosus (GoldFuss 1826 in GOLDFUSS \& MüNSTER 1826-1833), Echinocorys sp. und Galerites vulgaris (LESKE 1778) vorhanden. Zähne der Actinopterygiergattung Enchodus, sowie verschiedene Selachiertaxa, darunter Hexanchus microdon AgAssiz, 1843 in 18331843 und Carcharias aasenensis SIVERSON, 1992 sind ebenfalls häufig (LADWIG 2012, 2014). Auch ein Kieferfragment, welches CLAUSSEN \& LADWIG (1997) als Mosasaurierrest bestimmt haben, konnte in diesem Teil der Grube geborgen werden. 


\section{Fundgeschichte}

Am 9. März 1996 entdeckte der Zweitautor, Joachim Ladwig, beim Abbau der anstehenden Schreibkreide die hier beschriebenen Fossilien. Bereits bei der Bergung waren die Wirbelsäule und andere assoziierte Knochen erkennbar. Ein loser Brocken, der die Reste von drei Wirbelkörpern enthielt, wurde von J. Ladwig aufgesammelt und mitgenommen. Zwei Wochen darauf konnten weitere Teile, darunter ein großer Gastrolith (Länge ca. $47 \mathrm{~mm}, \mathrm{Abb} .3$ ) geborgen werden (letzterer befindet sich noch im Besitz von J. Ladwig).

Parallel kontaktierte der Finder das Geologische Institut der Universität Hamburg, durch deren Vermittlung einige Wochen später ein weiterer Besuch der Fundstelle erfolgte, an dem der mittlerweile verstorbene Echiniden-Experten Dr. Max-Gotthard Schulz von der Universität Kiel teilnahm. Hierbei konnten jene Reste gesichert werden, die sich nun im Besitz der Universität Hamburg befinden. Andere Teile des Skeletts wurden von Sammlern geborgen und befinden sich in Privatbesitz (für weitere Details siehe MAISCH \& SPAETH 2004: 101). Inzwischen ist die Lokalität von größeren Schuttmengen bedeckt, aber es ist zu vermuten, dass weitere Reste noch immer an der Fundstelle vorhanden sind.

\section{Systematische Paläontologie}

Sauropterygia OWEN, 1860

Plesiosauria de BLAINVILLE, 1835

Elasmosauridae COPE, 1869

Elasmosauridae gen. et sp. indet.

\section{Material:}

Namu ES/kro 36084, unvollständige Wirbel, Phalangen und diverse unbestimmbare Knochenfragmente.

\section{Fundort:}

Schreibkreidegrube "Saturn", Kronsmoor, Kreis Steinburg, Schleswig-Holstein.

\section{Fundschicht:}

Mittleren Bereich der grimmensis-/granulosus-Zone, Kronsmoor Formation, oberes Obercampanium.

\section{Beschreibung:}

Zwei Blöcke enthalten die Reste von fünf Wirbelcentra (Abb. 4a, b), die aber größtenteils beschädigt sind. Das besser erhaltene Centrum besitzt eine quadratische Form und hat eine Länge von $105 \mathrm{~mm}$. Die Artikulationsflächen sind, soweit dies erkennbar ist, nur schwach konkav. Die Größe und Form der Wirbel korrespondiert mit den von $\mathrm{MAISCH} \&$ SPAETH (2004) beschriebenen posterioren Cervicalwirbeln und Thoracalwirbeln (= Pectoraloder anterioren Dorsalwirbeln) des Exemplars.

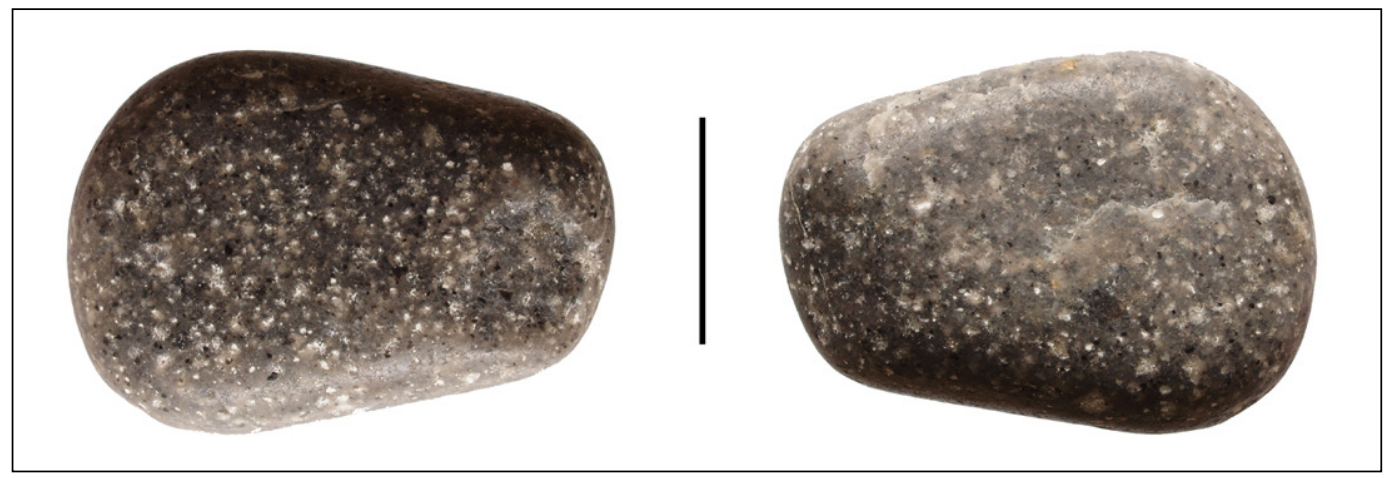

Abb. 3: Gastrolith des Kronsmoorer Elasmosauriers in der Sammlung von Joachim Ladwig.

Der Maßstab entspricht $2 \mathrm{~cm}$. 

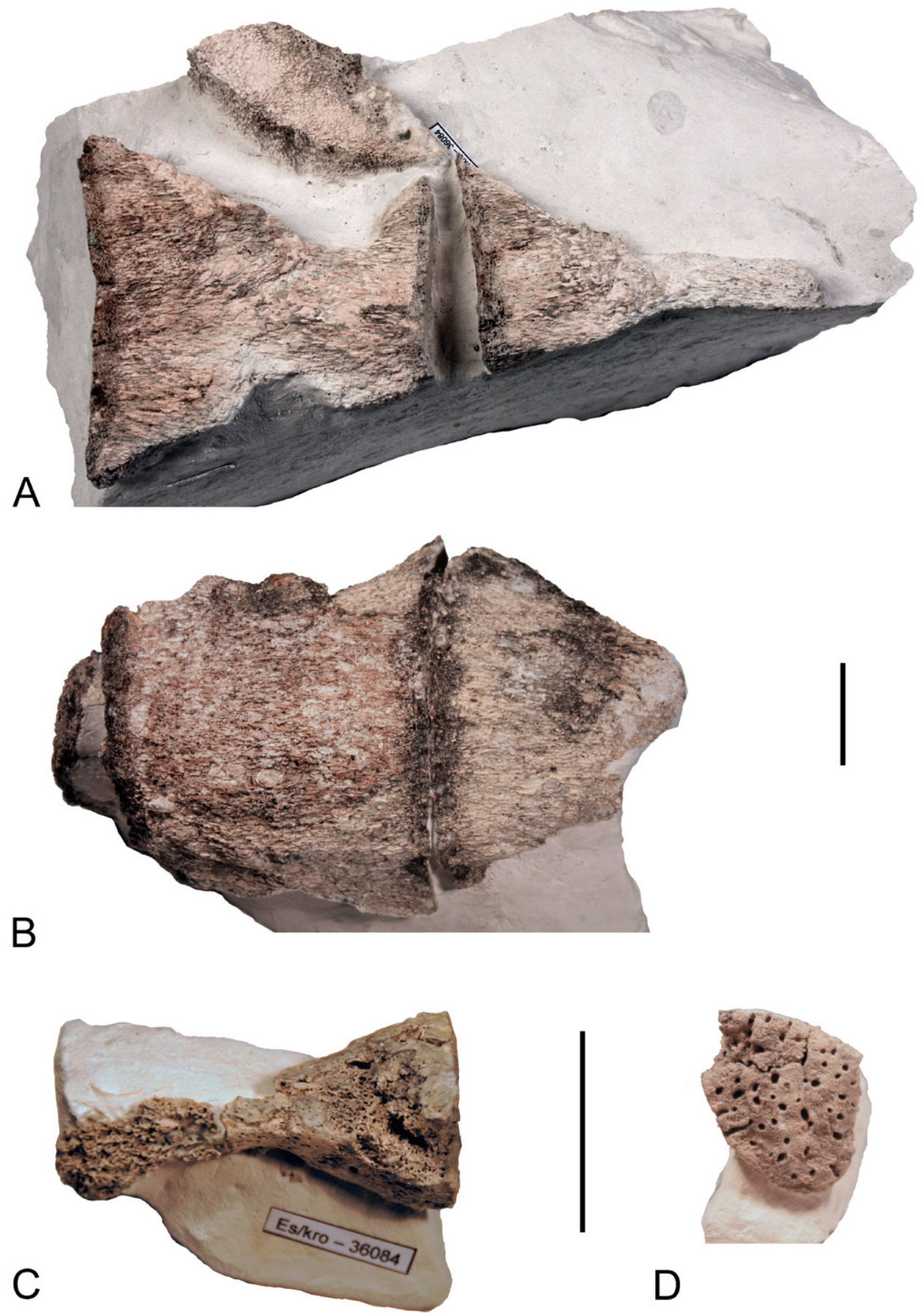

$\mathrm{D}$

Abb. 4: Elasmosauridae gen. et sp. indet., Namu ES/kro 36084. Unvollständige Wirbel (A, B). Phalange in (C) dorsaler oder ventraler und (D) proximaler oder distaler Ansicht. Der Maßstab entspricht $3 \mathrm{~cm}$. 
Eine genauere Bestimmung ist allerdings nicht möglich. Ein fast vollständiger Knochen kann als Phalange identifiziert werden (Abb. 4C, D) und zeigt die typische Sanduhrform, den diese Elemente bei Plesiosauriern besitzen. Das Artikulationsende einer zweiten Phalange liegt ebenfalls vor und bei beiden Stücken sind die Artikulationsflächen stark perforiert (Abb. 4d). Alle weiteren Teile von Namu ES/kro 36084 können nicht sicher bestimmt werden.

\section{Diskussion}

Die Funde in der Sammlung des Naturkunde-Museums Bielefeld ergänzen den spärlichen Nachweis der Elasmosauriden in der Oberkreide Deutschlands. Eine genauere taxonomische Zuordnung ist nur durch die anderen Teile des Individuums möglich, die von MAISCH \& SPAETH (2004) beschrieben wurden und sich in verschiedenen Sammlungen befinden. Maisch \& SPAETH (2004) schlußfolgerten, dass der Kronsmoorer Fund als Elasmosauridae gen. et sp. indet. bestimmt werden kann, der den nordamerikanischen Formen Elasmosaurus platyurus COPE 1868 und Alzadasaurus pembertoni Welles \& Bump 1949 am ähnlichsten ist.

Derguterhaltene Zahn (siehe MAISCH \& SPAETH 2004, Tafel 1, Abb. 1) zeigt typische Merkmale der Elasmosauriden, darunter das Fehlen von kräftigen Schmelzleisten und den an der Basis ovalen Querschnitt der Krone (siehe Diskussion in Ketchum \& Benson 2010, SACHS et al. 2017a). Ein von MaISCH \& SPAETH (2004) beschriebener amphicoeler (M. Maisch, pers. Mittl. 07.2017) Cervicalwirbel aus der Sammlung von M. Möller (siehe MaIsCH \& SPAETH 2004, Tafel 1, Abb. 2), der vermutlich aus dem vorderen Bereich des mittleren Halsabschnitts stammt, war etwa 1,7 Mal so lang wie breit (siehe MAISCH \& SPAETH 2004, S. 104). Bei den meisten Elasmosauriergattungen sind die mittleren Cervicalwirbel nicht, oder nur unwesentlich länger als breit (siehe z. B. Diskussion in Otero, 2016). Deutlich verlängerte mittlere Cervicalwirbel finden sich bisher nur bei drei validen Gattungen: Styxosaurus spp. (siehe Otero 2016, Welles 1952), Albertonectes vanderveldei KUBO, MITCHELL \& Henderson 2012 (siehe Kubo et al. 2012) und Elasmosaurus platyurus (siehe SACHS 2005, SACHS et al. 2013). Sie alle stammen aus dem Campanium Nordamerikas. Speziell zwei dieser Gattungen zeichnen sich durch einen extrem langen Hals aus, der bei Elasmosaurus aus 73 und bei Albertonectes aus 75 Wirbeln bestand. OtERo (2016) bezeichnet mittlere Halswirbel, die zwischen $2 / 3$ und doppelt so lang wie breit sind als "can-shaped" (dosenförmig) und betrachtet diese Wirbelform als ein diagnostisches Merkmal der Mitglieder der von ihm etablierten Unterfamilie Styxosaurinae. Dies konnte jedoch in einer neueren Studie nicht bestätigt werden (siehe SerRatos et al. 2017). Bei dem Holotypus von Styxosaurus snowii (WILliston 1890) liegt nur ein Teil des Halses vor, der die vorderen sowie einige mittlere Cervicalwirbel umfasst. Die Centra zeichnen sich dadurch aus dass sie deutlich länger als hoch sind und fast flache Artikulationsflächen besitzen (SACHS et al., eingereicht). Die Breite kann für die meisten Wirbel des Holotypus von Styxosaurus snowii nicht ermittelt werden, weil diese in eine Gipsmasse eingelassen wurden (S.Sachs, pers. Obs. November 2015). CARPENTER (1999) stellte ein weiteres Exemplar zu Styxosaurus snowii, welches zuvor als Alzadasaurus pembertoni beschrieben wurde und das auch MaIsCH \& SPAETH (2004) zum Vergleich herangezogen haben. Bei diesem Exemplar sind die Centra im vorderen Teil des mittleren Halses in ähnlicher Weise verlängert wie bei dem Kronsmoorer Fund (siehe WeLLES \& Bump 1949, Tab. 2). Der Holotypus von Styxosaurus snowii unterscheidet sich somit von dem mittleren Halswirbel aus Kronsmoor durch das Fehlen von konkaven Artikulationsflächen. Für den Holotypus von Alzadasaurus pembertoni liegt diese Information in der Literatur nicht vor. Falls jedoch CARPENTER (1999) mit seiner Zuordnung recht hat, ist zu erwarten, dass die mittleren Cervicalwirbel auch 
bei diesem Exemplar platycoel sind. Weniger stark verlängert (im Verhältnis zur Breite) als der Kronsmoorer Wirbel sind die mittleren Cervicalwirbel von Styxosaurus browni WeLLES 1952 (siehe Otero, 2016 Tab. 4) und Albertonectes vanderveldei (allerdings wurden von KuBo et al. (2012, Supplementary Table S2A) nur für wenige Wirbel Messwerte gegeben). Die vorderen und mittleren Halswirbel von Elasmosaurus platyurus sind hingegen z.T. deutlich länger als breit (siehe SACHS, 2005 Tab. 2) und sie besitzen ebenfalls konkave Artikulationsflächen (SACHS et al. 2013).

Zusammenfassend kann also gesagt werden, dass (basierend auf den vorliegenden Daten) Elasmosaurus platyurus die größte Übereinstimmung zeigt. Dennoch genügen die vorhandenen Merkmale nicht, um den Kronsmoorer Elasmosaurier zweifelsfrei dieser Gattung zuzuordnen. Da eine transatlantische Verbreitung von Elasmosauriern bereits beschrieben wurde (SACHS \& KEAR 2017) wäre es allerdings denkbar.

\section{Danksagung}

Der Erstautor dankt Herrn Joachim Ladwig (Norderbarup), dass er die hier beschriebenen Funde dem Naturkunde-Museum Bielefeld übergeben hat. Weiterer Dank gilt Dr. Michael Maisch (Tübingen), der hilfreiche Informationen zu dem von ihm beschriebenen Teil des Skelettes gab. Das namu dankt allen Besuchern, die für die Nachpräparation des Materials gespendet haben.

\section{Literatur}

AgASSIZ, L. (1833-1843): Recherches sur les Poissons Fossiles, 5 vols. Neuchâtel et Soleure: Petitpierre, 1420 pp.

Carpenter, K. (1999): Revision of North American elasmosaurs from the Cretaceous of the Western Interior. - Paludicola 2: 148-173.

Claussen, M. \& LadwiG, J. (1997): Das Kieferfragment eines Mosasauriers aus dem Obercampan von Kronsmoor. - Der Geschiebesammler 30(3): 127-130.

COPE, E. D. (1868): Remarks on a new enaliosaurian, Elasmosaurus platyurus. - Proceedings of the Academy of Natural Sciences Philadelphia 20: 92-93.

COPE, E. D. (1869): Synopsis of the extinct Batrachia and Reptilia of North America. Part 1. Transactions of the American Philosophical Society 14: 1-235.

de Blainville, H. D. (1835): Description de quelques espèces de reptiles de la Californie, précédée de l'analyse d'un système général d'Erpétologie et d'Amphibiologie. Nouvelles Annales du Muséum (national) d'Histoire Naturelle de Paris 4: 233-296.

Foth, C., KalBE, J., KautZ, R. (2011): First evidence of Elasmosauridae (Reptilia: Sauropterygia) in an erratic boulder of Campanian age originating from southern Sweden or the adjacent Baltic Sea area. - Zitteliana A51: 285-290.

FreRICHS, U. (1994): Elasmosaurus sp. aus dem Untercampan von Höver. Eine Funddokumentation. - Arbeitskreis Paläontologie Hannover 22(2): 33-42.

Goldfuss, G. A., Münster, G. (1826-1833): Petrefacta Germaniæ - Abbildungen und Beschreibungen der Petrefacten Deutsch- 
lands und der angränzenden Länder. Erster Theil. (Arnz \& Comp.) Düsseldorf: 1-252.

Ketchum, H. F., Benson, R. B. J. (2010): Global interrelationships of Plesiosauria (Reptilia, Sauropterygia) and the pivotal role of taxon sampling in determining the outcome of phylogenetic analyses. - Biological Reviews 85: 361-392.

Kubo, T., Mitchell, M. T., Henderson, D. M. (2012): Albertonectes vanderveldei, a new elasmosaur (Reptilia, Sauropterygia) from the Upper Cretaceous of Alberta. - Journal of Vertebrate Paleontology 32(3): 557-572.

LADWIG, J. (1997): Mosasaurierreste aus Schleswig Holstein. - Fossilien 1997(6): 358-362.

LADWIG, J. (2012): Zu welcher Spezies gehören die Zähne der Gattung Hexanchus (Chondrichthyes, Hexanchiformes) aus der nordwestdeutschen Oberkreide? - Arbeitskreis Paläontologie Hannover, 40(2): 42-56.

LADWIG, J. (2014): Zähne der Hai-Gattung Carcharias aus dem oberen Campanium der Schreibkreidegrube "Saturn“ in Kronsmoor (Schleswig-Holstein). - Arbeitskreis Paläontologie Hannover, 42(1): 1-11.

LESKE, N. G. (1778): Additamenta ad J. T. Kleinii naturalem dispositionem Echinodermatum, Gleditschiana, Lipsiae, 214 S.

Lomax, D. R. \& WAHL, W. R. (2013): A new specimen of the elasmosaurid plesiosaur Zarafasaura oceanis from the Upper Cretaceous (Maastrichtian) of Morocco. - Paludicola 9: 97-109.

MaISCH, M. W., SPAETH, C. (2004): Skelettreste und Gastrolithen eines Elasmosauriers (Sauropterygia) aus der Schreibkreidegrube von Kronsmoor bei Lägerdorf (SchleswigHolstein). - Geologisches Jahrbuch A157: 99-119.
NieTSCH, H. (1921): Die irregularen Echiniden der pommerschen Kreide. - Abhandlungen aus dem geologisch-palaeontologischen Institut der Universität Greifswald 2, 247 S.

Otero, R. (2016): Taxonomic reassessment of Hydralmosaurus as Styxosaurus: new insights on the elasmosaurid neck evolution throughout the Cretaceous. - PeerJ 4: e1777.

OWEN, R. (1860): On the orders of fossil and recent Reptilia, and their distribution in time. - Reports of the British Association for the Advancement of Science, London 29: 153-166.

SACHS, S. (2005): Redescription of Elasmosaurus platyurus Cope 1868 (Plesiosauria: Elasmosauridae) from the Upper Cretaceous (lower Campanian) of Kansas, USA. - Paludicola 5: 92-106.

SACHS, S. (2011): Ein elasmosaurider Plesiosaurier aus dem unteren Campan (Oberkreide) von Sehnde-Höver (Landkreis Hannover). Arbeitskreis Paläontologie Hannover 39: 12-19.

SACHS, S. \& KeAR, B. P. (2015): Fossil Focus: Elasmosaurs. - Palaeontology Online 5: 1-8.

SACHS, S. \& KEAR, B. P. (2017): Redescription of the elasmosaurid plesiosaurian Libonectes atlasense from the Upper Cretaceous of Morocco. - Cretaceous Research 74: 205-222.

SACHS, S., KeAR, B. P. \& Everhart, M. J. (2013): Revised vertebral count in the "longestnecked vertebrate" Elasmosaurus platyurus Cope 1868, and clarification of the cervicaldorsal transition in Plesiosauria. - PLoS ONE 8, e70877.

Sachs, S., Wilmsen, M., Knüppe, J., Hornung, J. J., KEAR, B. P. (2016a): Kreide-Fossilien in Sachsen. Teil 15. Reptilien. - Geologica Saxonica 62: 169-179. 
Sachs, S., Wilmsen, M., Knüppe, J., Hornung, J. J., KEAR, B. P. (2017a): Cenomanian-Turonian marine amniote remains from the Saxonian Cretaceous Basin of Germany. - Geological Magazine 154(2): 237-246.

Sachs, S., Hornung, J. J., Scheer, U. (2017b): Mosasaurid and plesiosaurian remains from marginal facies of the lower Campanian (Upper Cretaceous) Bottrop and Vaals formations of western Germany. - Cretaceous Research.

Sachs, S., Lindiren, J., Kear, B. P. (eingereicht): Reassessment of the Styxosaurus snowii (Williston, 1890) type specimen and its implications for the elasmosaurid plesiosaurian clade Styxosaurinae. - Alcheringa.

Schulz, M.-G. (1985): Die Evolution der Echiniden-Gattung Galerites im Campan und Maastricht Norddeutschlands. - Geologisches Jahrbuch A80: 93 S.

Serratos, D. J., Druckenmiller, P., Benson, R. B. (2017): A new elasmosaurid (Sauropterygia, Plesiosauria) from the Bearpaw Shale (Late Cretaceous, Maastrichtian) of Montana demonstrates multiple evolutionary reductions of neck length within Elasmosauridae. Journal of Vertebrate Paleontology 37(2): e1278608.

SivERSON, M. (1992): Biology, dental morphology and taxonomy of lamniform sharks from the Campanian of the Kristianstad Basin, Sweden. - Palaeontology 35(3): 519-554

Welles, S. P. (1943): Elasmosaurid plesiosaurs with a description of new material from California and Colorado. - University of California Publications in Geological Sciences 13: $125-215$.
Welles, S. P. (1952): A review of the North American Cretaceous elasmosaurs. - University of California Publications in Geological Sciences 29: 47-143.

Welles, S. P. \& Bump, J. (1949): Alzadasaurus pembertoni, a new elasmosaur from the Upper Cretaceous of South Dakota. - Journal of Paleontology 23: 521-535.

WILLISTON, S. W. (1890): Structure of the plesiosaurian skull. - Science 16: 262. 